\title{
Entanglement entropy for integer quantum Hall effect in two and higher dimensions
}

\author{
Dimitra Karabali@* \\ Physics and Astronomy Department, Lehman College, CUNY, Bronx, New York 10468, USA
}

(Received 26 June 2020; accepted 8 July 2020; published 21 July 2020)

\begin{abstract}
We analyze the entanglement entropy, in real space, for the higher-dimensional integer quantum Hall effect on $\mathbb{C P}^{k}$ (any even dimension) for Abelian and non-Abelian magnetic background fields. In the case of $\nu=1$ we perform a semiclassical calculation which gives the entropy as proportional to the phase-space area. This exhibits a certain universality in the sense that the proportionality constant is the same for any dimension and for any background, Abelian or non-Abelian. We also point out some distinct features in the profiles of the eigenfunctions of the two-point correlator that underline the difference in the value of entropies between $\nu=1$ and higher Landau levels.
\end{abstract}

DOI: $10.1103 /$ PhysRevD.102.025016

\section{INTRODUCTION}

Entanglement has been used to explore properties of quantum states in a variety of condensed matter systems. Typically a system is divided into two subsystems and the entanglement is calculated in terms of the von Neumann entropy of the reduced density matrix of one of the subsystems. For gapped two-dimensional systems, the leading order contribution to the entanglement entropy is proportional to the perimeter of the boundary separating the two subsystems, in particular $S=c L+\gamma+\mathcal{O}(1 / L)$, where $L$ is the length of the boundary, $c$ is a nonuniversal coefficient and $\gamma$ is a universal quantity called topological entanglement entropy [1].

Of particular interest among two-dimensional gapped systems are the quantum Hall systems whose entanglement entropy has been widely studied under different partitions. Entanglement calculations have been used to characterize various topologically ordered phases and further extract information on the edge properties of such systems [2-8]. This is one of the key motivations for the study of entanglement entropy in the context of quantum Hall effect. For a real-space partition it was found that $\gamma=0$ for fully filled integer quantum Hall states and nonzero for fractional quantum Hall states. The entanglement entropy in the case of integer quantum Hall states is amenable to analytical calculations due to the fact that the many-body ground state is given in terms of free fermions. The area-law entropy

\footnotetext{
*dimitra.karabali@lehman.cuny.edu
}

Published by the American Physical Society under the terms of the Creative Commons Attribution 4.0 International license. Further distribution of this work must maintain attribution to the author(s) and the published article's title, journal citation, and DOI. Funded by SCOAP. behavior for the two-dimensional integer quantum Hall effect (QHE) was studied in different geometries analytically for $\nu=1$ and numerically up to $\nu=5$ in [2] and the coefficient $c$ was identified in these cases. A general mathematical theorem on the entropy area law for $\nu=1$ is shown in [9].

In this paper we present a detailed analysis of the entanglement entropy in the case of higher-dimensional integer quantum Hall effect (any even dimension), in particular quantum Hall effect on $\mathbb{C P}^{k}[10-12]$. For $k=1$ this reduces to the well-known case of QHE on $S^{2}$ where the magnetic field is created by a monopole at the center [13]. The formulation of QHE on $\mathbb{C P}^{k}$ for $k>1$ displays two interesting features: higher dimensionality and the possibility of introducing both Abelian and non-Abelian magnetic fields. In the latter case one deals with a manybody system of free fermions with internal degrees of freedom which is amenable to analytical calculations.

The paper is organized as follows. In Sec. II we give a brief description of the integer quantum Hall effect on $\mathbb{C} \mathbb{P}^{k}$, the corresponding spectrum and the Landau level wave functions. In Sec. III we focus on the lowest Landau level and derive analytical expressions for the entropy for (a) arbitrary $k$ and Abelian $U(1)$ magnetic field and (b) $k=2$ for $U(1) \times S U(2)$ non-Abelian magnetic field with fermions in the triplet representation. The entropy is expressed in terms of the eigenvalues of the two-point correlation function. We perform a semiclassical calculation for the entropy and find that the area law as expressed in terms of a "phase-space" area has the same coefficient $c$ for $\nu=1$ for any dimension and any Abelian or nonAbelian background. In Sec. IV we derive analytical expressions for the eigenvalues of the two-point correlator for the first Landau level and the $\nu=2$ quantum Hall system on $S^{2}$ and comment on how the different profiles 
account for the different values of $c$ in each case. We end with a short discussion.

\section{QUANTUM HALL EFFECT ON $\mathbb{C P}^{k}$}

In this section we will give a brief description of the Landau level states and wave functions for the quantum Hall effect on $\mathbb{C P}^{k}$, following a group theoretic analysis developed in [10-12]. $\mathbb{C P}^{k}$ is a $2 k$-dimensional manifold which can be thought of as a coset space,

$$
\mathbb{C P}^{k}=\frac{S U(k+1)}{U(k)}
$$

The Landau wave functions can be obtained as functions of $S U(k+1)$ with specific transformation properties under the $U(k)$ subgroup. A basis for such functions is given by the so-called Wigner $\mathcal{D}$-functions, which are the matrices corresponding to the group elements in the unitary irreducible representations, namely

$$
\mathcal{D}_{L, R}^{J}(g)=\left\langle J, l_{A}|g| J, r_{A}\right\rangle
$$

where $J$ denotes the representation and $l_{A}, r_{A}$ stand for two sets of quantum numbers specifying the states within the representation. On an element $g \in S U(k+1)$, we can define left and right $S U(k+1)$ actions by

$$
\hat{L}_{A} g=T_{A} g, \quad \hat{R}_{A} g=g T_{A}
$$

where $T_{A}$ are the $S U(k+1)$ generators in the representation to which $g$ belongs. The left transformations correspond to magnetic translations. There are $2 k$ right generators of $S U(k+1)$ which are not in $U(k)$; these can be separated into $T_{+i}, i=1,2 \ldots, k$, which are of the raising type and $T_{-i}$ which are of the lowering type. These generate translations while $U(k)$ generates rotations at a point. The covariant derivatives on $\mathbb{C P}^{k}$ are given by

$$
\mathcal{D}_{ \pm i}=i \frac{\hat{R}_{ \pm i}}{r}
$$

where $r$ can be thought of as the radius of $\mathbb{C P}^{k}$. This is consistent with the fact that the commutator of covariant derivatives is the magnetic field. The commutators of $\hat{R}_{+i}$ and $\hat{R}_{-i}$ are in the Lie algebra of $U(k)$; in the case of $\mathbb{C P}^{k}$ these correspond to constant magnetic fields. In particular we can specify the background field by specifying the right action of $U(k)$ on the wave functions

$$
\begin{gathered}
\hat{R}_{a} \Psi_{m ; \alpha}^{J}(g)=\left(T_{a}^{\tilde{J}}\right)_{\alpha \beta} \Psi_{m ; \beta}^{J}(g), \\
\hat{R}_{k^{2}+2 k} \Psi_{m ; \alpha}^{J}(g)=-\frac{n k}{\sqrt{2 k(k+1)}} \Psi_{m ; \alpha}^{J}(g)
\end{gathered}
$$

where the index $m=1, \ldots, \operatorname{dim} J$ represents the state within the $S U(k+1)$ representation $J$ and therefore counts the degeneracy of the Landau level. The first of these equations shows that the wave functions $\Psi_{m ; \alpha}^{J}$ transform, under right rotations, as a representation $\tilde{J}$ of $S U(k) .\left(T_{a}^{\tilde{J}}\right)_{\alpha \beta}$ are the representation matrices for the generators of $S U(k)$ in the representation $\tilde{J}$ and $n$ is an integer characterizing the Abelian part of the background field. $\alpha, \beta$ label states within the $S U(k)$ representation $\tilde{J}$ [which is itself contained in the representation $J$ of $S U(k+1)]$. The index $\alpha$ in the wave functions $\Psi_{m ; \alpha}^{J}(g)$ characterizes the non-Abelian charge of the underlying fermion fields.

In terms of $\mathcal{D}$-functions, the correctly normalized wave functions are given by

$$
\Psi_{m ; \alpha}^{J}(g)=\sqrt{N}\langle J, m|g| J, \alpha, n\rangle=\sqrt{N} \mathcal{D}_{m ; \alpha}^{J}(g)
$$

where $N=\operatorname{dim} J$ and the following orthogonality theorem has been used

$$
\int d \mu(g) \mathcal{D}_{m ; \alpha}^{* J}(g) \mathcal{D}_{m^{\prime} ; \alpha^{\prime}}^{J}(g)=\frac{\delta_{m m^{\prime}} \delta_{\alpha \alpha^{\prime}}}{N}
$$

where $d \mu(g)$ is the Haar measure on $S U(k+1)$ normalized to unity.

In the absence of a confining potential, the Hamiltonian $H$ for the Landau problem is proportional to the covariant Laplacian on $\mathbb{C P}^{k}$, namely

$$
H \Psi=-\frac{1}{4 m}\left(\mathcal{D}_{+i} \mathcal{D}_{-i}+\mathcal{D}_{-i} \mathcal{D}_{+i}\right) \Psi
$$

which apart from additive constants can be reduced to the form $\sum_{i} \hat{R}_{+i} \hat{R}_{-i}$. Thus the lowest Landau level wave functions satisfy the holomorphicity condition

$$
\hat{R}_{-i} \Psi=0
$$

The conditions (5), (6) and (10) completely fix the representation $J$ and therefore the degeneracy of the lowest Landau level.

First we consider the lowest Landau wave functions for the case of an Abelian background magnetic field. In that case the state $|J, n\rangle$ corresponds to the singlet representation of $S U(k) \in S U(k+1)$ with a $U(1)$ charge proportional to $n$ as specified in (6), namely $R_{3}=-n / 2$. These can be thought of as the coherent states for $\mathbb{C P}^{k}$, written explicitly in terms of complex coordinates,

$$
\begin{aligned}
\Psi_{i_{1} i_{2} \cdots i_{k}} & \left.=\sqrt{N}\left[\frac{n !}{i_{1} ! i_{2} ! \ldots i_{k} !(n-s) !}\right]\right]^{\frac{1}{2}} \frac{z_{1}^{i_{1}} z_{2}^{i_{2}} \cdots z_{k}^{i_{k}}}{(1+\bar{z} \cdot z)^{\frac{n}{2}}}, \\
s & =i_{1}+i_{2}+\cdots+i_{k}, \quad 0 \leq i_{i} \leq n, \quad 0 \leq s \leq n
\end{aligned}
$$


where $z$ 's are holomorphic coordinates, $z=x+i y$. These wave functions form a symmetric, rank $n$ representation $J$ of $S U(k+1)$. The dimension of this representation, which is also the lowest Landau level (LLL) degeneracy, is

$$
N=\operatorname{dim} J=\frac{(n+k) !}{n ! k !} .
$$

The volume element for $\mathbb{C \mathbb { P } ^ { k }}$ is

$$
d \mu=\frac{k !}{\pi^{k}} \frac{d^{2} z_{1} \cdots d^{2} z_{k}}{(1+\bar{z} \cdot z)^{k+1}} .
$$

We have chosen the normalization such that the total volume, $\int d \mu$, is 1 .

In the case of a $U(1) \times S U(k)$ non-Abelian background, it is convenient to label the irreducible representation of $S U(k+1)_{R}$ by $\left(p+l, q+l^{\prime}\right)$ corresponding to the tensor [11]

$$
\mathcal{T}_{b_{1} \ldots b_{p} \delta_{1} \ldots \delta_{l}}^{a_{1} \ldots a_{q} \gamma_{1} \ldots \gamma_{l^{\prime}}} \equiv \mathcal{T}_{p, l}^{q, l^{\prime}}
$$

where $p, q$ indicate $U(1)$ indices and $l, l^{\prime}$ indicate $S U(k)$ indices; namely $a$ 's and $b$ 's take the value $(k+1)$ and $\gamma$ 's and $\delta$ 's take values $1, \ldots, k$.

The right hypercharge corresponding to (6) is

$$
\sqrt{2 k(k+1)} R_{k^{2}+2 k}=-k(p-q)+l-l^{\prime}=-n k .
$$

The fact that $n$ has to be an integer implies that $\left(l-l^{\prime}\right) / k$ is an integer, thus constraining the possible $S U(k)_{R}$ representations $\tilde{J}$.

Further, as explained in detail in [11], the lowest Landau level states correspond to $q=0, l=0$. So the LLL states we consider correspond to the tensor $\mathcal{T}_{p}^{l^{\prime}}$, where $p=n-\frac{l^{\prime}}{k}$ and $l^{\prime}=j k, j=1,2, \ldots$

\section{ENTANGLEMENT ENTROPY FOR $\nu=1$}

In order to calculate the entanglement entropy we divide the system into two regions, $D$ and its complementary $D^{C}$, and define the reduced density matrix

$$
\rho_{D}=\operatorname{Tr}_{D^{c}}|G S\rangle\langle G S|
$$

where $|G S\rangle$ is the many-body ground state of the system, $|G S\rangle=\prod_{m} c_{m}^{\dagger}|0\rangle$.

The entanglement entropy is defined as

$$
S=-\operatorname{Tr} \rho_{D} \log \rho_{D}
$$

and is a measure of the entanglement between the two subsystems.

We choose $D$ to be the spherically symmetric region of $\mathbb{C P}^{k}$ satisfying $z \cdot \bar{z} \leq R^{2}$. For $\mathbb{C P}^{1} \sim S^{2}$, this region is a polar cap centered around the north pole and bounded by a latitude angle $\theta$, with $R=\tan \theta / 2$ via stereographic projection.

The reduced density matrix $\rho_{D}$ is related to the two-point correlator $C\left(r, r^{\prime}\right)[2,5]$

$$
C\left(r, r^{\prime}\right)=\left\langle G S\left|\Psi^{\dagger}(r) \Psi\left(r^{\prime}\right)\right| G S\right\rangle
$$

where $\Psi(r)$ is the electron field operator and $r, r^{\prime}$ are restricted to be inside the domain $D$. In particular, the reduced matrix $\rho_{D}$ can be written as a $2^{N} \times 2^{N}$ matrix of a block diagonal form

$$
\rho_{D}=\bigotimes_{m} \operatorname{diag}\left(\lambda_{m}, 1-\lambda_{m}\right)
$$

where $\lambda_{m}$ are the eigenvalues of the two-point correlator $C\left(r, r^{\prime}\right)$. The entanglement entropy is then given by

$$
S=-\sum_{m=1}^{N}\left[\lambda_{m} \log \lambda_{m}+\left(1-\lambda_{m}\right) \log \left(1-\lambda_{m}\right)\right] .
$$

For $\nu=1$,

$$
\Psi(r)=\sum_{m=1}^{N} \Psi_{m}(z) c_{m}
$$

where $\Psi_{m}(z)$ are the holomorphic wave functions discussed in the previous section and the index $m$ counts the degeneracy of the lowest Landau level. Using (18) and (21) we find that

$$
C\left(r, r^{\prime}\right)=\sum_{m=1}^{N} \Psi_{m}^{*}(z) \Psi_{m}\left(z^{\prime}\right)
$$

where $z, z^{\prime}$ are restricted to be inside the domain $D$. The diagonalization of $C\left(z, z^{\prime}\right)$ gives the result

$$
\begin{aligned}
\int_{D} C\left(r, r^{\prime}\right) \Psi_{l}^{*}\left(z^{\prime}\right) d \mu\left(z^{\prime}\right) & =\sum_{m=0}^{N} \Psi_{m}^{*}(z) \int_{D} \Psi_{m}\left(z^{\prime}\right) \Psi_{l}^{*}\left(z^{\prime}\right) d \mu\left(z^{\prime}\right) \\
& =\lambda_{l} \Psi_{l}^{*}(z)
\end{aligned}
$$

where

$$
\lambda_{l}=\int_{D}\left|\Psi_{l}\right|^{2} d \mu
$$

The second line in (23) is due to the fact that since $\Psi_{m}(z)$ are holomorphic functions given in (11) the angular integration over the spherically symmetric region $D$ will give zero unless $m=l$, namely $\int_{D} \Psi_{m}\left(z^{\prime}\right) \Psi_{l}^{*}\left(z^{\prime}\right) d \mu\left(z^{\prime}\right)=\delta_{l m} \lambda_{l}$.

We now proceed to calculate the eigenvalues $\lambda$ and subsequently the entanglement entropy for the case of an Abelian and non-Abelian magnetic field backgrounds. 


\section{A. $\mathbb{C P}^{k}$ and Abelian magnetic field background}

The lowest Landau level wave functions for $\mathbb{C} \mathbb{P}^{k}$ in the case of an Abelian background magnetic field are given in (11). The corresponding eigenvalues of the two-point correlator are

$$
\begin{aligned}
\lambda_{i_{1} i_{2} \cdots i_{k}}= & \int_{D} d \mu \Psi_{i_{1} i_{2} \cdots i_{k}}^{*}(r) \Psi_{i_{1} i_{2} \cdots i_{k}}(r) \\
= & \frac{(n+k) !}{i_{1} ! i_{2} ! \cdots i_{k} !(n-s) !} \int_{D} \frac{\left(\bar{z}_{1} z_{1}\right)^{i_{1}}\left(\bar{z}_{2} z_{2}\right)^{i_{2}} \cdots\left(\bar{z}_{k} z_{k}\right)^{i_{k}}}{(1+\bar{z} \cdot z)^{n+k+1}} \\
& \times \frac{d^{2} z_{1} \cdots d^{2} z_{k}}{\pi^{k}}
\end{aligned}
$$

where $s=i_{1}+i_{2}+\cdots+i_{k}$. We perform the angular integration using the parametrization $z_{i}=x_{2 i-1}+i x_{2 i}$, where

$$
\begin{aligned}
x_{1}= & \rho \cos \left(\phi_{1}\right), \\
x_{2}= & \rho \sin \left(\phi_{1}\right) \cos \left(\phi_{2}\right), \\
& \cdots \\
x_{2 k-1}= & \rho \sin \left(\phi_{1}\right) \sin \left(\phi_{2}\right) \cdots \sin \left(\phi_{2 k-2}\right) \cos \left(\phi_{2 k-1}\right), \\
x_{2 k}= & \rho \sin \left(\phi_{1}\right) \sin \left(\phi_{2}\right) \cdots \sin \left(\phi_{2 k-1}\right)
\end{aligned}
$$

and $0 \leq \phi_{1}, \phi_{2}, \ldots, \phi_{2 k-2} \leq \pi, 0 \leq \phi_{2 k-1} \leq 2 \pi$. Using the fact that in terms of this parametrization

$$
\begin{aligned}
d^{2} z_{1} \cdots d^{2} z_{k}= & \rho^{2 k-1} d \rho \sin \left(\phi_{1}\right)^{2 k-2} d \phi_{1} \sin \left(\phi_{2}\right)^{2 k-3} d \phi_{2} \cdots \\
& \times \sin \left(\phi_{2 k-2}\right) d \phi_{2 k-2} d \phi_{2 k-1}
\end{aligned}
$$

and

$$
\int_{0}^{\pi}(\sin \phi)^{2 i} d \phi=\sqrt{\pi} \frac{\Gamma\left(i+\frac{1}{2}\right)}{\Gamma(i+1)}
$$

we find, after doing the angular integrations, that

$\lambda_{i_{1} i_{2} \cdots i_{k}} \equiv \lambda_{s}=\frac{(n+k) !}{(n-s) !(s+k-1) !} \int_{0}^{R^{2}} \frac{x^{s+k-1}}{(1+x)^{n+k+1}} d x$.

For each value of $s=i_{1}+i_{2}+\cdots+i_{k}$, the eigenvalue $\lambda_{s}$ has a degeneracy $d_{s}=(s+k-1) ! / s !(k-1) !$.

The expression for the entanglement entropy is then

$S=\sum_{s=0}^{n} \frac{(s+k-1) !}{s !(k-1) !}\left[-\lambda_{s} \log \lambda_{s}-\left(1-\lambda_{s}\right) \log \left(1-\lambda_{s}\right)\right]$.

We will now evaluate the entanglement entropy using a semiclassical approximation and relate this to the area of the boundary of $D$. This is possible when the $U(1)$ charge $n$, which controls the dimensionality of the lowest Landau Hilbert space, becomes very large.
Making a change of variables to $t=x /(1+x)$, the expression for the eigenvalues $\lambda$ in (29) can be written as

$$
\begin{aligned}
\lambda_{s} & =\frac{(n+k) !}{(s+k-1) !(n-s) !} \int_{0}^{t_{0}} d t t^{s+k-1}(1-t)^{n-s} \\
& =\frac{(n+k) !}{(s+k-1) !(n-s) !} B\left(t_{0} ; s+k, n-s+1\right)
\end{aligned}
$$

where $t_{0}=R^{2} /\left(1+R^{2}\right)$ and $B\left(z ; m_{1}, m_{2}\right)$ is the incomplete beta function. For large $n$ this is amenable to a semiclassical calculation as shown in [14]. We will follow that derivation here. Equation (31) can be written as

$$
\begin{aligned}
\lambda_{s} & =\frac{(n+k) !}{(s+k-1) !(n-s) !} \int_{0}^{t_{0}} d t e^{F(t),} \\
F(t) & =(s+k-1) \log t+(n-s) \log (1-t) .
\end{aligned}
$$

The maximum of $F(t)$ occurs at $t^{*}=(s+k-1) /(n+k-1)$. Expanding $F(t)$ around $t^{*}$ we find that $e^{F}$ becomes a Gaussian function centered around $t^{*}$. In fact,

$$
\left.\frac{d^{2} F}{d t^{2}}\right|_{t^{*}}=-\frac{(n+k-1)^{3}}{(n-s)(s+k-1)}
$$

which implies that the width of the Gaussian is very narrow for all $s$. For small $s$ the center of the Gaussian, $t^{*} \sim 0$, falls within the range of integration and we find that $\lambda_{s} \sim 1$. For large $s \sim n$ the center of the Gaussian, $t^{*} \sim 1>t_{0}$, falls outside the range of integration and therefore $\lambda_{s} \sim 0$. The middle of the transition occurs at $s^{*}$ such that $t^{*}=t_{0}$, namely

$t^{*}=\frac{s^{*}+k-1}{n+k-1}=t_{0} \Rightarrow \begin{aligned} & s^{*}=t_{0}(n+k-1)-(k-1) \\ & n-s^{*}=(n+k-1)\left(1-t_{0}\right) .\end{aligned}$

Expanding $F(t)$ around $t_{0}$ in (35) we find

$$
\begin{aligned}
F(t)= & (n+k-1)\left[t_{0} \log t_{0}+\left(1-t_{0}\right) \log \left(1-t_{0}\right)\right] \\
& -\frac{(n+k-1)}{2 t_{0}\left(1-t_{0}\right)}\left(t-t_{0}\right)^{2}+\cdots .
\end{aligned}
$$

Using this expression we find that for large $n$,

$$
\begin{aligned}
\int_{0}^{t_{0}} e^{F(t)} & \sim e^{F\left(t_{0}\right)} \int_{0}^{t_{0}} \exp \left[-\frac{(n+k-1)}{2 t_{0}\left(1-t_{0}\right)}\left(t-t_{0}\right)^{2}\right] \\
& =e^{F\left(t_{0}\right)} \sqrt{\frac{\pi t_{0}\left(1-t_{0}\right)}{2(n+k-1)}} .
\end{aligned}
$$

Substituting this in (32) and using Stirling's formula $n !=\sqrt{2 \pi n}(n / e)^{n}$, we find that 


$$
\lambda_{s_{*}} \approx \frac{n+k}{2(n+k-1)} \rightarrow \frac{1}{2}
$$

for any $t_{0}$. The value of $t_{0}$ is controlled by $R$, which characterizes the size of the spherical domain $D$ and the above calculation shows that $\lambda_{s}$ is significantly different from 0 or 1 only for $s$ such that the corresponding wave functions are localized very near the boundary of the entangling surface.

For large $n$ we can define a variable $y=s /(n+k-1)$, $0 \leq y \leq 1$, and consider $\lambda$ as a continuous function of $y$. From what we have seen before $\lambda \rightarrow 1$ as $y \rightarrow 0, \lambda \rightarrow 0$ as $y \rightarrow 1$ and $\lambda \rightarrow 1 / 2$ as $y \rightarrow s^{*} /(n+k-1)=t_{0}$. In deriving a semiclassical expression for the entanglement entropy we will also need to calculate the derivative of $\lambda$ at the transition region, namely $\left.\frac{d \lambda}{d y}\right|_{y=t_{0}}$. For that we have to calculate the difference $\lambda_{s^{*}+i}-\lambda_{s^{*}}$. For $s=s^{*}+i$, the maximum of $F(t)$ occurs at

$$
t_{1}=\frac{s_{*}+k-1}{n+k-1}+\frac{i}{n+k-1}=t_{0}+\epsilon,
$$

where $\epsilon=i /(n+k-1) \ll 1$ for small $i$ and large $n$. We now expand $F$ in (32) around $t_{1}$, but because the peak has been shifted beyond the upper limit of integration, (36) will give an extra contribution proportional to $\epsilon$ for small $\epsilon$,

$$
\int_{0}^{t_{0}} \exp \left[-\frac{(n+k-1)}{2 t_{1}\left(1-t_{1}\right)}\left(t-t_{1}\right)^{2}\right] \sim \sqrt{\frac{\pi t_{0}\left(1-t_{0}\right)}{2(n+k-1)}}-\epsilon .
$$

Using Stirling's formula and taking $\epsilon \rightarrow 0$ we find

$$
\begin{aligned}
\left.\frac{d \lambda}{d y}\right|_{y=t_{0}} & =\lim _{\epsilon \rightarrow 0} \frac{\lambda_{s^{*}+i}-\lambda_{s^{*}}}{\epsilon} \\
& \sim-\sqrt{\frac{n+k-1}{2 \pi t_{0}\left(1-t_{0}\right)}}+\mathcal{O}\left(\frac{1}{\sqrt{n}}\right) .
\end{aligned}
$$

Figures 1 and 2 show plots of $\lambda_{s}$ for different values of $k$ and $t_{0}=R^{2} /\left(1+R^{2}\right)$.

We found in (30) that the expression for the entanglement entropy is

$$
\begin{aligned}
S & =\sum_{s=0}^{n} \frac{(s+k-1) !}{s !(k-1) !} H_{s}, \\
H_{s} & =-\lambda_{s} \log \lambda_{s}-\left(1-\lambda_{s}\right) \log \left(1-\lambda_{s}\right) .
\end{aligned}
$$

It is clear from the Figs. 1 and 2 that $H_{s}$ is nonzero only for values of $s$ very near the transition region where $\lambda_{s^{*}}=1 / 2$. We can then expand $H(\lambda(y))$ around the value $\lambda=1 / 2$,

$$
H(\lambda(y))=H\left(t_{0}\right)+\frac{1}{2} \frac{d^{2} H}{d y^{2}}\left(y-t_{0}\right)^{2}+\cdots
$$

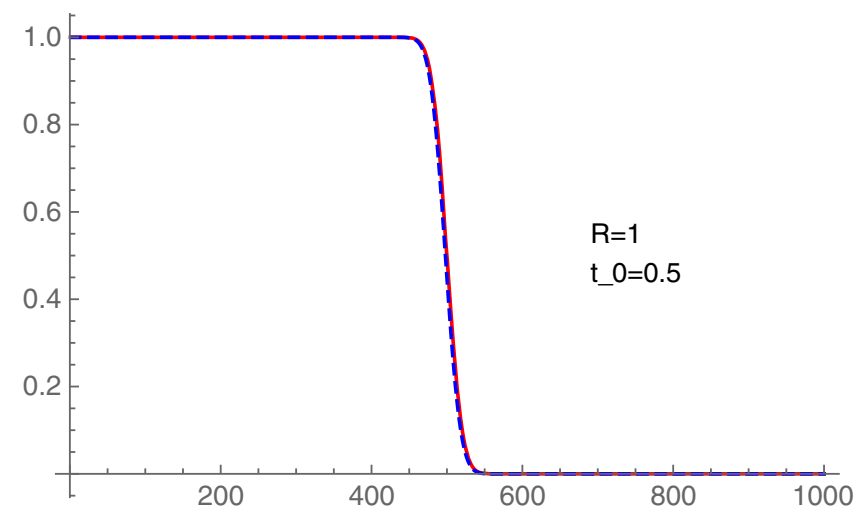

FIG. 1. Plots of $\lambda_{s}$ as a function of $s$ for $k=1$ (red), $k=5$ (blue dashed) and $n=1000$ and $R=1$.

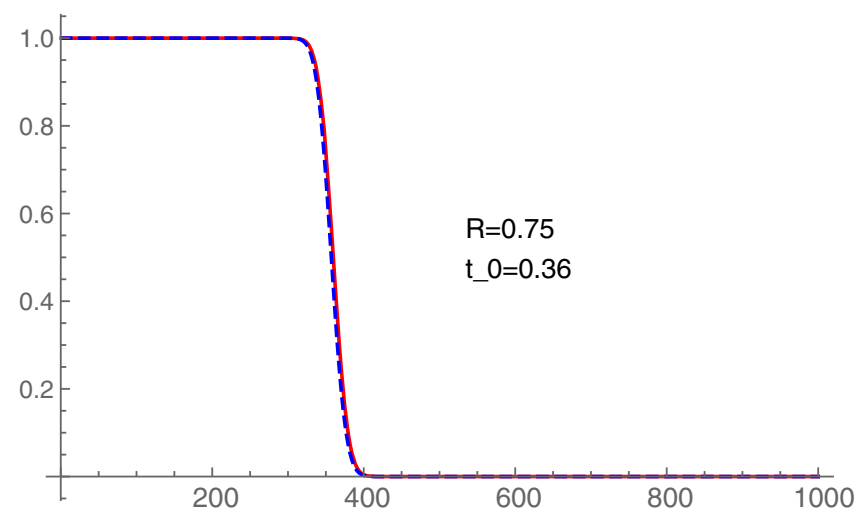

FIG. 2. Plots of $\lambda_{s}$ as a function of $s$ for $k=1$ (red), $k=5$ (blue dashed) and $n=1000$ and $R=0.75$.

where

$$
\begin{aligned}
\left.\frac{d H}{d y}\right|_{y=t_{0}} & =\left.\frac{d H}{d \lambda} \frac{d \lambda}{d y}\right|_{\lambda=1 / 2}=0, \\
\left.\frac{d^{2} H}{d^{2} y}\right|_{y=t_{0}} & =\frac{d H}{d \lambda} \frac{d^{2} \lambda}{d y^{2}}+\left.\frac{d^{2} H}{d \lambda^{2}}\left(\frac{d \lambda}{d y}\right)^{2}\right|_{\lambda=1 / 2} \\
& =-\frac{4(n+k-1)}{2 \pi t_{0}\left(1-t_{0}\right)}
\end{aligned}
$$

Since $H$ has a narrow support around $\lambda=1 / 2$ it can be approximated by the Gaussian

$$
\begin{aligned}
H(y) & =H_{0} \exp \left[\frac{1}{2} \frac{d^{2} H}{d \lambda^{2}}\left(\frac{d \lambda}{d y}\right)^{2}\left(y-t_{0}\right)^{2}\right] \\
& =\log 2 e^{-\frac{(n+k-1)}{\pi \log 2 t_{0}\left(1-t_{0}\right)}\left(y-t_{0}\right)^{2}} .
\end{aligned}
$$

We can rescale to $s=y(n+k-1)$ and $s^{*}=t_{0}(n+k-1)-$ $(k-1)$ to obtain the semiclassical Gaussian approximation to $H_{s}$ as 


$$
\begin{aligned}
H_{s, k}= & \log 2 \exp \left[-\frac{1}{\pi \log 2 t_{0}\left(1-t_{0}\right)}\right. \\
& \left.\times \frac{\left(s+k-1-t_{0}(n+k-1)\right)^{2}}{n+k-1}\right] .
\end{aligned}
$$

Figures 3 and 4 show the validity of the Gaussian approximation by comparing (45) to the exact expression (41), (31).

We can now use (45) to analytically calculate the entropy in (41) for large $n$ by converting the sum into an integral over the variable $y$,

$$
\begin{aligned}
S & \sim n \frac{s^{* k-1}}{(k-1) !} \log 2 \int_{0}^{1} \exp \left[-\frac{(n+k-1)}{\pi \log 2 t_{0}\left(1-t_{0}\right)}\left(y-t_{0}\right)^{2}\right] d y \\
& \sim n^{k-\frac{1}{2}} \frac{t_{0}^{k-1}}{(k-1) !} \pi(\log 2)^{3 / 2} \sqrt{t_{0}\left(1-t_{0}\right)} \\
& \sim n^{k-\frac{1}{2}} \frac{\pi(\log 2)^{3 / 2}}{(k-1) !} \frac{R^{2 k-1}}{\left(1+R^{2}\right)^{k}} .
\end{aligned}
$$

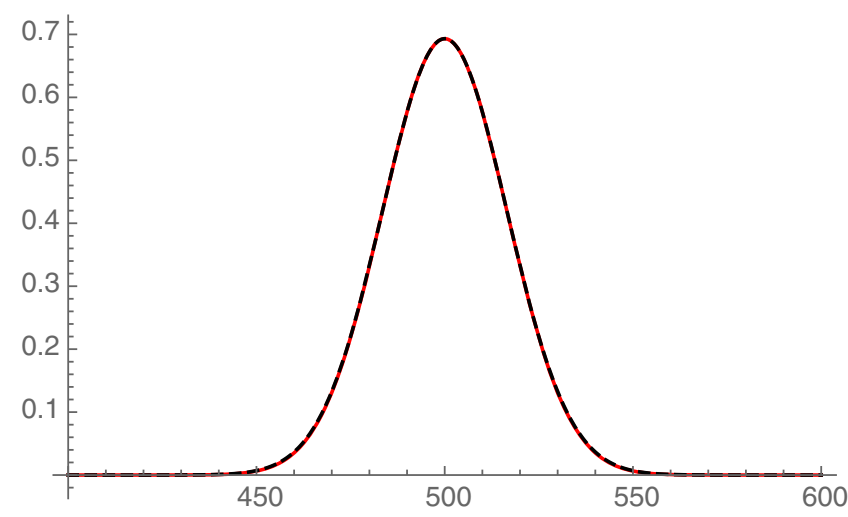

FIG. 3. Plots of $H_{s}$ exact (red) and Gaussian approximation (blue dashed) as a function of $s$ for $k=1$ and $n=1000$ and $R=1$.

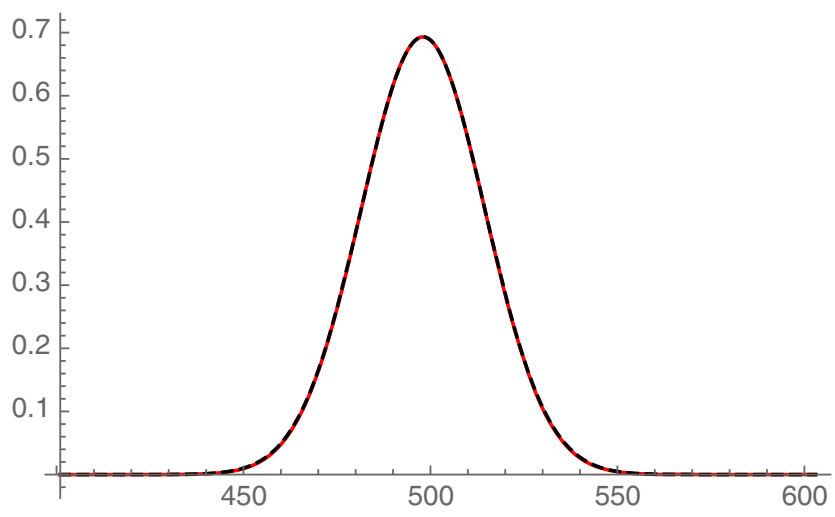

FIG. 4. Plots of $H_{s}$ exact (red) and Gaussian approximation (blue dashed) as a function of $s$ for $k=5$ and $n=1000$ and $R=1$.
The fact that the entropy is proportional to the entangling area $\frac{R^{2 k-1}}{\left(1+R^{2}\right)^{k}}$ has to do with the fact that only wave functions localized around the entanglement boundary with corresponding eigenvalues $\lambda \sim 1 / 2$ contribute to the entropy.

For $k=1$ this agrees with the result found in [2]. In the case of the QHE on the sphere the entangling surface is a circle of perimeter $L=2 \pi \sin \theta$, where, based on the stereographic projection $2 R /\left(1+R^{2}\right)=\sin \theta$. Scaling the radius of the entangling surface by $\sqrt{n / 2}$ (for QHE on $S^{2}$ the monopole charge, magnetic field and radius of the sphere are related by $n=2 B r^{2}$ ) we find the area law quoted in [2]

$$
S(k=1)=\frac{\sqrt{2}(\log 2)^{3 / 2}}{4} L=0.204 L .
$$

The normalized volume element (13), upon angular integration can be written in terms of the radial variable $\rho$ defined in (26)

$$
d \mu=\frac{k !}{\pi^{k}} \frac{d^{2} z_{1} \cdots d^{2} z_{k}}{(1+\bar{z} \cdot z)^{k+1}}=2 k \frac{\rho^{2 k-1}}{\left(1+\rho^{2}\right)^{k}} \frac{d \rho}{1+\rho^{2}}
$$

where $e_{\rho}=d \rho /\left(1+\rho^{2}\right)$ is the vierbein along the radial direction $\rho$. This defines the geometric area of the entangling surface (with volume normalized to 1 ) to be $A_{\text {geom }}=2 k \frac{R^{2 k-1}}{\left(1+R^{2}\right)^{k}}$. On the other hand the phase-space volume which is proportional to the degrees of freedom is $V_{\text {phase space }}=\frac{n^{k}}{k !} \int d \mu$. This then defines a phase-space surface area

$$
A_{\text {phase space }}=\frac{n^{k-\frac{1}{2}}}{k !} A_{\text {geom }}=2 \frac{n^{k-\frac{1}{2}}}{(k-1) !} \frac{R^{2 k-1}}{\left(1+R^{2}\right)^{k}} .
$$

Scaling the entanglement entropy in (46) in terms of this phase-space area we derive a universal expression valid in all dimensions, with a proportionality constant independent of $k$, namely

$$
S \sim \frac{\pi}{2}(\log 2)^{3 / 2} A_{\text {phase space }}
$$

\section{B. $\mathbb{C P} P^{2}$ and non-Abelian magnetic field background}

The derivation of the entanglement entropy in the case of a non-Abelian background magnetic field is more involved. As mentioned in Sec. II the LLL states form irreducible representations of $S U(k+1)$ of the form $\mathcal{T}_{p}^{l^{\prime}}$, where $p=n-\frac{l^{\prime}}{k}$ and $l^{\prime}=j k, j=1,2, \ldots$. We will elucidate the calculation of the entanglement entropy for the special case of $\mathbb{C} \mathbb{P}^{2}$ with a non-Abelian magnetic field $U(1) \times S U(2)$ for the lowest value of $l^{\prime}$, namely $l^{\prime}=k=2$ and $p=n-1$. The derivation for other values of $k$ and $l^{\prime}$ follows similar ideas. The dimension of this representation and therefore the degeneracy of the corresponding LLL is [11] 


$$
N=\frac{3 n(n+3)}{2}
$$

In identifying the corresponding wave functions we consider the states $\langle m|\hat{g}| w\rangle$, where the states on the right are of the form $\mathcal{T}_{p}^{2}$ with two up indices and transforming as the $\tilde{J}=1$ triplet representation of $S U(2) \in S U(3)$. [Since the lowest allowed value for $l^{\prime}$ is 2, based on (15) and following comments, the doublet representation is not allowed for $\mathbb{C P}^{2}$.] The corresponding group elements in the appropriate representation can be constructed in terms of products of elements of the $3 \times 3$ matrix $g$ which forms the fundamental representation of $S U(3)$ and its conjugate $g^{*}$. We need $p$ copies of $g$ and two copies of $g^{*}$ to match the structure of the $\mathcal{T}_{p}^{2}$ representation. In terms of these matrices, choosing the state $|w\rangle$ as explained above, we get

$$
\left\langle i_{1} i_{2} ; j_{1} \cdots j_{p}|\hat{g}| 3 \cdots 3 ; \alpha \beta\right\rangle \sim g^{* i_{1} \alpha} g^{* i_{2} \beta} g_{j_{1} 3} \cdots g_{j_{p} 3}
$$

where $i, j=1,2,3$ and $\alpha, \beta=1,2$. Within (52) there are three distinct series, each one forming an $S U(2)$ multiplet under the left transformations, and all of them together comprising the full $\nu=1$ lowest Landau level $S U(3)$ representation. The three such series are of the form:

Series 1

$$
\Psi_{(\alpha \beta)}^{(1)} \sim g^{* 3 \alpha} g^{* 3 \beta}\left(g_{13}\right)^{l}\left(g_{23}\right)^{m-l}\left(g_{33}\right)^{n-1-m}
$$

where $l=0, \ldots, m$ and $m=0, \ldots, n-1$. For each $m,(53)$ form an $S U(2)$ left representation with $j=m / 2$. There are $\sum_{0}^{n-1}(m+1)=n(n+1) / 2$ such states.

Series 2

$\Psi_{(\alpha \beta)}^{(2)} \sim\left(g^{* \gamma \alpha} g^{* 3 \beta}+g^{* 3 \alpha} g^{* \gamma \beta}\right)\left(g_{13}\right)^{l}\left(g_{23}\right)^{m-l}\left(g_{33}\right)^{n-1-m}$.

For each $m,(54)$ form an $S U(2)$ left representation with $j=(m+1) / 2$. There are $\sum_{0}^{n-1}(m+2)=n(n+3) / 2$ such states.

\section{Series 3}

$\Psi_{(\alpha \beta)}^{(3)} \sim\left(g^{* \gamma \alpha} g^{* \delta \beta}+g^{* \gamma \beta} g^{* \delta \alpha}\right)\left(g_{13}\right)^{l}\left(g_{23}\right)^{m-l}\left(g_{33}\right)^{n-1-m}$.

For each $m$, (55) form an $S U(2)$ left representation with $j=(m / 2)+1$. There are $\sum_{0}^{n-1}(m+3)=n(n+5) / 2$ such states.

Considering all three series together, the total number of states are $N=3 n(n+3) / 2$ confirming the result in (51). We now proceed to normalize the above wave functions. In doing so we will use the fact that the elements $g_{i 3}$ can be written in terms of the complex coordinates parametrizing $\mathbb{C P}^{2}$, namely

$$
\begin{aligned}
g_{\alpha 3} & =\frac{z_{\alpha}}{\sqrt{1+\bar{z} \cdot z}}, \quad \alpha=1,2, \\
g_{33} & =\frac{1}{\sqrt{1+\bar{z} \cdot z}}
\end{aligned}
$$

where $\bar{z} \cdot z=\bar{z}_{1} z_{1}+\bar{z}_{1} z_{1}$.

States, with the correct normalization within each series, can be explicitly constructed by starting with the highest weight state and applying the lowering operator $J_{-}$as follows:

$$
\begin{aligned}
J_{-} g_{13} & =g_{23}, & J_{-} g_{23} & =0, \\
J_{-} g^{* 2 i} & =-g^{* 1 i}, & J_{-} g^{* 1 i} & =0 .
\end{aligned}
$$

\section{Series 1 normalization}

The highest weight state within this $S U(2)$ multiplet is the state of the form

$$
|J, J\rangle=C_{1} g^{* 3 \alpha} g^{* 3 \beta}\left(g_{13}\right)^{m}\left(g_{33}\right)^{n-1-m}
$$

where $C_{1}$ is the normalization factor to be determined. The rest of the states are obtained by applying the lowering operator $J_{-}$whose action is indicated in (57), namely

$$
|J, J-l\rangle=C_{1} g^{* 3 \alpha} g^{* 3 \beta}\left(g_{13}\right)^{m-l}\left(g_{23}\right)^{l}\left(g_{33}\right)^{n-1-m}
$$

where $l=0,1, \ldots, m$. Using (58) and the fact that $g^{\dagger} g=1$ we find that

$\sum_{\alpha \beta} \Psi_{\alpha \beta}^{*(1)} \Psi_{\alpha \beta}^{(1)}=\left|C_{1}\right|^{2}\left[1-\frac{1}{1+\bar{z} \cdot z}\right]^{2} \frac{\left(\bar{z}_{1} z_{1}\right)^{m-l}\left(\bar{z}_{2} z_{2}\right)^{l}}{(1+\bar{z} \cdot z)^{n-1}}$.

The $\mathbb{C P}^{2}$ volume element is $d \mu=\frac{2}{\pi^{2}} \frac{d^{2} z_{1} d^{2} z_{2}}{(1+\bar{z} \cdot z)^{3}}$. Using the relation

$$
\int \frac{\left(\bar{z}_{1} z_{1}\right)^{l}\left(\bar{z}_{2} z_{2}\right)^{m}}{(1+\bar{z} \cdot z)^{n+1}} d \mu=2 \frac{l ! m !(n+1-l-m) !}{(n+3) !}
$$

we find that the correctly normalized wave function is of the form

$$
\begin{aligned}
\Psi_{(l, m ; \alpha \beta)}^{(1)}= & \sqrt{\frac{(n+3) !}{2 l !(m-l) !(m+2)(m+3)(n-1-m) !}} \\
& \times g^{* 3 \alpha} g^{* 3 \beta}\left(g_{13}\right)^{m-l}\left(g_{23}\right)^{l}\left(g_{33}\right)^{n-1-m}
\end{aligned}
$$

where $l=0,1, \ldots, m$.

\section{Series 2 normalization}

The highest weight state within this $S U(2)$ multiplet is given by

$|J, J\rangle=C_{2}\left(g^{* 2 \alpha} g^{* 3 \beta}+g^{* 3 \alpha} g^{* 2 \beta}\right)\left(g_{13}\right)^{m}\left(g_{33}\right)^{n-1-m}$.

Acting with the lowering operator $J_{-}$as before we obtain the rest of the states which are of the form 


$$
\begin{aligned}
|J, J-l\rangle= & C_{2}\left[-l \frac{m !}{(m-l+1) !}\left(g^{* 1 \alpha} g^{* 3 \beta}+g^{* 3 \alpha} g^{* 1 \beta}\right)\left(g_{13}\right)^{m-l+1}\left(g_{23}\right)^{l-1}\right. \\
& \left.+\frac{m !}{(m-l) !}\left(g^{* 2 \alpha} g^{* 3 \beta}+g^{* 3 \alpha} g^{* 2 \beta}\right)\left(g_{13}\right)^{m-l}\left(g_{23}\right)^{l}\right]\left(g_{33}\right)^{n-1-m}
\end{aligned}
$$

where $l=0,1, \ldots, m+1$. Using again the relation (61) we find the normalized wave functions to be of the form

$$
\begin{aligned}
\Psi_{(l, m ; \alpha \beta)}^{(2)}= & \sqrt{\frac{(n+3) !}{4 l !(m-l+1) !(m+1)(m+3)(n+1)(n-1-m) !}} \\
& \times\left[-l\left(g^{* 1 \alpha} g^{* 3 \beta}+g^{* 3 \alpha} g^{* 1 \beta}\right)\left(g_{13}\right)^{m-l+1}\left(g_{23}\right)^{l-1}\right. \\
& \left.+(m-l+1)\left(g^{* 2 \alpha} g^{* 3 \beta}+g^{* 3 \alpha} g^{* 2 \beta}\right)\left(g_{13}\right)^{m-l}\left(g_{23}\right)^{l}\right]\left(g_{33}\right)^{n-1-m},
\end{aligned}
$$

$l=0,1, \ldots, m+1$.

\section{Series 3 normalization}

The highest weight state in series 3 is given by

$$
|J, J\rangle=C_{3} g^{* 2 \alpha} g^{* 2 \beta}\left(g_{13}\right)^{m}\left(g_{33}\right)^{n-1-m} .
$$

Every other state in this multiplet is constructed as before by applying $l$ times the lower operator $J_{-}$, producing

$$
\begin{aligned}
|J, J-l\rangle= & C_{3}\left[\frac{m !}{(m-l) !} g^{* 2 \alpha} g^{* 2 \beta}\left(g_{23}\right)^{l}\left(g_{13}\right)^{m-l}\right. \\
& -l \frac{m !}{(m-l+1) !}\left(g^{* 1 \alpha} g^{* 2 \beta}+g^{* 2 \alpha} g^{* 1 \beta}\right)\left(g_{13}\right)^{m-l+1}\left(g_{23}\right)^{l-1} \\
& \left.+l(l-1) \frac{m !}{(m-l+2) !} g^{* 1 \alpha}\left(g_{13}\right)^{m-l+2}\left(g_{23}\right)^{l-2}\right]\left(g_{33}\right)^{n-1-m}
\end{aligned}
$$

where $l=0,1, \ldots, m+2$. Using (61) we find the normalized wave functions to be of the form

$$
\begin{aligned}
\Psi_{(l, m ; \alpha \beta)}^{(3)}= & \sqrt{\frac{(n+3) !}{2 l !(m-l+2) !(m+1)(m+2)(n+1)(n+2)(n-1-m) !}} \\
& \times\left[l(l-1) g^{* 1 \alpha} g^{* 1 \beta}\left(g_{13}\right)^{m-l+2}\left(g_{23}\right)^{l-2}-l(m-l+2)\left(g^{* 1 \alpha} g^{* 2 \beta}+g^{* 2 \alpha} g^{* 1 \beta}\right)\left(g_{13}\right)^{m-l+1}\left(g_{23}\right)^{l-1}\right. \\
& \left.+(m-l+1)(m-l+2) g^{* 2 \alpha} g^{* 2 \beta}\left(g_{13}\right)^{m-l}\left(g_{23}\right)^{l}\right]\left(g_{33}\right)^{n-1-m},
\end{aligned}
$$

$l=0,1, \ldots, m+2$.

The analog of the two-point correlator (22) carries now non-Abelian indices and is given by

$$
C_{a b}\left(r, r^{\prime}\right)=\sum_{A} \Psi_{A ; a}^{*}(r) \Psi_{A ; b}\left(r^{\prime}\right)
$$

where we have denoted collectively the left indices by $A=(l, m)$ and the right (non-Abelian) indices by $a=(\alpha \beta)$. The diagonalization of $C_{a b}\left(r, r^{\prime}\right)$ gives

$$
\sum_{b} \int C_{a b}\left(r, r^{\prime}\right) \Psi_{A ; b}^{*}\left(r^{\prime}\right) d \mu^{\prime}=\lambda \Psi_{A ; a}^{*}(r)
$$

where the eigenvalues $\lambda$ are defined

$$
\lambda=\sum_{a} \int_{D} \Psi_{A ; a}^{*}(r) \Psi_{A, a}(r) d \mu(r) .
$$

In deriving this we used the fact the wave functions (62), (65), (68) are orthogonal to each other.

We find that there are three distinct expressions for $\lambda$ 's, one for each of the $S U(2)$ multiplets described above. After performing the angular integration in (71) using (27) and (28) we find 


$$
\begin{aligned}
& \lambda_{s}^{(1)}=\frac{(n+3) !}{(s+3) !(n-1-s) !} \int^{R^{2}} \frac{d x}{(1+x)^{n+4}} x^{s+3}, \\
& \lambda_{s}^{(2)}=\frac{(n+3) !}{(s+2) !(n-1-s) !(n+1)} \int^{R^{2}} \frac{d x}{(1+x)^{n+4}}\left[x^{s+2}+\frac{s+1}{s+3} x^{s+3}\right], \\
& \lambda_{s}^{(3)}=\frac{(n+3) !}{(s+1) !(n-1-s) !(n+1)(n+2)} \int^{R^{2}} \frac{d x}{(1+x)^{n+4}}\left[x^{s+1}+\frac{2(s+1)}{s+2} x^{s+2}+\frac{s+1}{s+3} x^{s+3}\right]
\end{aligned}
$$

with the corresponding degeneracy $s+1, s+2$ and $s+3$. As $R^{2} \rightarrow \infty, \lambda_{s}^{(I)} \rightarrow 1$ confirming the correct normalization for the wave functions.

The expression for the entanglement entropy for the non-Abelian lowest Landau level states for $\mathbb{C P}^{2}$ can now be written as

$$
\begin{aligned}
S & =\sum_{s=0}^{n-1}\left[(s+1) H_{s}^{(1)}+(s+2) H_{s}^{(2)}+(s+3) H_{s}^{(3)}\right] \\
H_{s}^{(I)} & =-\lambda_{s}^{(I)} \log \lambda_{s}^{(I)}-\left(1-\lambda_{s}^{(I)}\right) \log \left(1-\lambda_{s}^{(I)}\right)
\end{aligned}
$$

with the $\lambda^{(I)}$ 's given in (72).

We will now show that $\lambda^{(I)}$ 's in (72) can be related to the Abelian ones in (31) making a semiclassical calculation of (73) similar to the Abelian case. Making a change of variables to $t=x /(1+x)$ as before we find that (72) can be written as

$$
\begin{aligned}
& \lambda_{s}^{(1)}=\frac{(n+3) !}{(s+3) !(n-s-1) !} \int_{0}^{t_{0}} d t t^{s+3}(1-t)^{n-s-1}, \\
& \lambda_{s}^{(2)}=\frac{(n+3) !}{(s+2) !(n-s-1) !(n+1)} \int_{0}^{t_{0}} d t\left[t^{s+2}(1-t)^{n-m-1}-\frac{2 t^{s+3}(1-t)^{n-s-1}}{s+3}\right], \\
& \lambda_{s}^{(3)}=\frac{(n+3) !}{(s+1) !(n-s-1) !(n+1)(n+2)} \int_{0}^{t_{0}} d t\left[t^{s+1}(1-t)^{n-s-1}-\frac{2 t^{s+2}(1-t)^{n-s-1}}{s+2}+\frac{2 t^{s+3}(1-t)^{n-s-1}}{(s+2)(s+3)}\right] .
\end{aligned}
$$

Comparing these to the Abelian $\mathbb{C P}^{k}$ values (31) which we denote by $\lambda^{(\mathrm{Ab})}$ we find the following relations,

$$
\begin{aligned}
& \lambda_{s, k=2}^{(1)}=\lambda_{s+1, k=3}^{(\mathrm{Ab})}, \\
& \lambda_{s, k=2}^{(2)}=\frac{n+3}{n+1} \lambda_{s+1, k=2}^{(\mathrm{Ab})}-\frac{2}{n+1} \lambda_{s+1, k=3}^{(\mathrm{Ab})}, \\
& \lambda_{s, k=2}^{(3)}=\frac{n+3}{n+1} \lambda_{s+1, k=1}^{(\mathrm{Ab})}-\frac{2(n+3)}{(n+1)(n+2)} \lambda_{s+1, k=2}^{(\mathrm{Ab})}+\frac{2}{(n+1)(n+2)} \lambda_{s+1, k=3}^{(\mathrm{Ab})} .
\end{aligned}
$$

At the large- $n$ limit the non-Abelian eigenvalues $\lambda^{(I)}$ for $k=2$ coincide with the Abelian ones for $k=1,2,3$ correspondingly,

$$
\lambda_{s, k=2}^{(1)}=\lambda_{s+1, k=3}^{(\mathrm{Ab})}, \quad \lambda_{s, k=2}^{(2)} \sim \lambda_{s+1, k=2}^{(\mathrm{Ab})}, \quad \lambda_{s, k=2}^{(3)} \sim \lambda_{s+1, k=1}^{(\mathrm{Ab})} .
$$

Similarly, at the large- $n$ limit, the non-Abelian entropy (73) becomes a multiple of the Abelian one in (46)

$$
\begin{aligned}
S & =\sum_{s=0}^{n-1}\left[(s+1) H_{s, k=2}^{(1)}+(s+2) H_{s, k=2}^{(2)}+(s+3) H_{s, k=2}^{(3)}\right] \\
& \sim \sum_{s=0}^{n-1}\left[(s+1) H_{s+1, k=3}^{(\mathrm{Ab})}+(s+2) H_{s+1, k=2}^{(\mathrm{Ab})}+(s+3) H_{s+1, k=1}^{(\mathrm{Ab})}\right] \\
& \sim 3 n^{3 / 2} \pi(\log 2)^{3 / 2} \frac{R^{3}}{\left(1+R^{2}\right)^{2}}=3 S^{(\mathrm{Ab})}
\end{aligned}
$$


The overall factor of 3 relating the non-Abelian entanglement entropy to the Abelian one above has to do with the fact that each lowest Landau state is an $S U(2)$ triplet, $\operatorname{dim} \tilde{J}=3$. Although the calculation of the entropy in the case of a non-Abelian background was explicitly done for $\mathbb{C P}^{2}$ and the triplet representation, one expects a more general statement to hold. In the large- $n$ limit the degeneracy of the LLL in a case of a non-Abelian background is $[10,11]$

$$
N \sim \operatorname{dim} \tilde{J} \frac{n^{k}}{k !}
$$

The corresponding phase-space volume in this case is $V_{\text {phase space }}=\operatorname{dim} \tilde{J} \frac{n^{k}}{k !} \int d \mu$ and the corresponding phasespace surface area is

$$
\begin{aligned}
A_{\text {phase space }} & =n^{\frac{2 k-1}{2}} \frac{\operatorname{dim} \tilde{J}}{k !} A_{\text {geom }} \\
& =n^{k-\frac{1}{2}} \frac{2 \operatorname{dim} \tilde{J}}{(k-1) !\left(1+R^{2}\right)^{k}} .
\end{aligned}
$$

Expressed in terms of the phase-space surface area the overall coefficient in the expression for the entanglement entropy is the same for any Abelian or non-Abelian background at large $n$,

$$
S \sim \frac{\pi}{2}(\log 2)^{3 / 2} A_{\text {phase space }}
$$

\section{HIGHER LANDAU LEVELS}

In this section we will consider the entropy for higher Landau levels focusing in particular on some of the differences in the behavior of the eigenvalues $\lambda$ between the lowest Landau level $q=0$, the first excited Landau level $q=1$ and the case of $\nu=2$ where both levels are filled. We will only consider the $k=1$ case, QHE on the sphere. Similar features apply for higher $k$. form

The wave functions for the $q$ th Landau level are of the

$$
\Psi_{m}^{J}(g)=\sqrt{N}\langle J, m|g| J, n\rangle
$$

where $J=n / 2+q$ and $\operatorname{dim} J=n+2 q+1$. The state $|J, n\rangle$ is not the lowest weight state of the $J$ representation. The lowest weight state is the LLL state with $n \rightarrow n+2 q$. The $q=1$ states can therefore be generated by the action of $\hat{R}_{+}$on the LLL states with $n \rightarrow n+2$. In the case of the sphere the representation of the $\hat{R}_{i}$-operators is of the form

$$
\begin{aligned}
& \hat{R}_{+}=-\epsilon_{\alpha \beta} u_{\alpha}^{*} \frac{\partial}{\partial u_{\beta}}, \quad \hat{R}_{-}=\epsilon_{\alpha \beta} u_{\alpha} \frac{\partial}{\partial u_{\beta}^{*}}, \\
& \hat{R}_{3}=\sum_{\alpha} \frac{1}{2}\left[-u_{\alpha} \frac{\partial}{\partial u_{\alpha}}+u_{\alpha}^{*} \frac{\partial}{\partial u_{\alpha}^{*}}\right]
\end{aligned}
$$

where

$$
u_{\alpha}=\frac{1}{\sqrt{1+\bar{z} z}}\left(\begin{array}{l}
z \\
1
\end{array}\right)
$$

The $\hat{R}$-operators satisfy the $S U(2)$ algebra

$$
\left[\hat{R}_{+}, \hat{R}_{-}\right]=2 \hat{R}_{3} .
$$

Based on the argument above the correctly normalized wave functions of the $q=1$ Landau level are

$$
\begin{aligned}
\Psi_{s}^{q=1}= & \sqrt{n+3} \sqrt{\frac{(n+1) !}{s !(n+2-s) !}} \hat{R}_{+}\left(u_{1}^{s} u_{2}^{n+2-s}\right) \\
= & \sqrt{n+3} \sqrt{\frac{(n+1) !}{s !(n+2-s) !}} \\
& \times\left[\frac{-(n+2) \bar{z} z^{s}}{\sqrt{(1+z \bar{z})^{n+2}}}+\frac{s z^{s-1}}{\sqrt{(1+z \bar{z})^{n}}}\right] .
\end{aligned}
$$

The corresponding eigenvalues of the two-point correlator are now of the form

$$
\begin{aligned}
\lambda_{s}^{(q=1)}= & \frac{(n+3) !}{(n+2) s !(n+2-s) !} \\
& \times \int^{R^{2}} d x \frac{x^{s-1}}{(1+x)^{n+4}}[(n+2-s) x-s]^{2} .
\end{aligned}
$$

Changing variables to $x=\frac{t}{(1-t)}$ as before we can rewrite the eigenvalues as

$$
\lambda_{s}^{(q=1)}=\frac{(n+3) !(n+2)}{s !(n+2-s) !} \int_{0}^{t_{0}} d t t^{s-1}(1-t)^{n-s+1}\left[t-\frac{s}{n+2}\right]^{2} .
$$

The eigenvalue $\lambda_{s}^{(q=1)}$ as a function of $s$ is similar to $\lambda_{s}^{(q=0)}$ away from the transition region, but it displays a distinct steplike pattern around the transition $s=t_{0}(n+2)$, as shown in Figs. 5 and 6. The reason for this has to do with the fact that the wave functions (85) have a node. Since they are generated by the action of $R_{+}$on the LLL wave functions of monopole charge $n+2$ they are necessarily orthogonal to them. Since the LLL wave functions are nonzero and have no node, orthogonality requires that the first level Landau wave functions must have a node. Higher Landau level wave functions acquire more nodes and one 


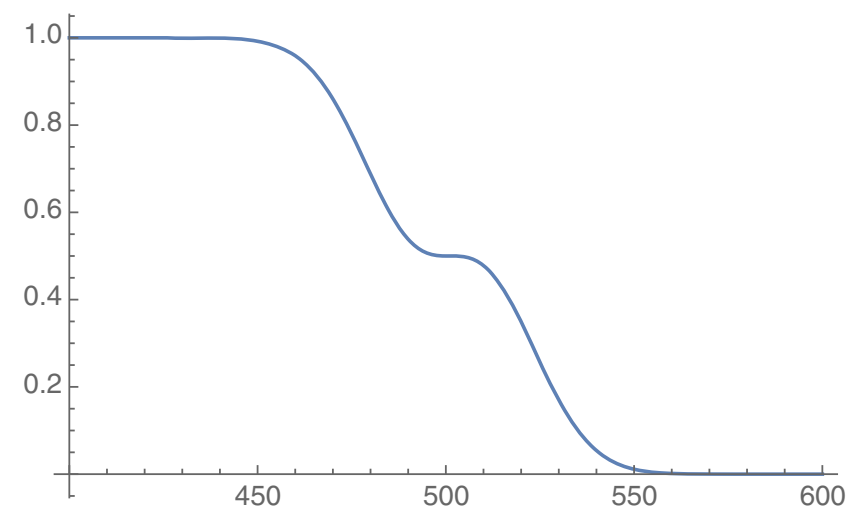

FIG. 5. Plot of $\lambda_{s}^{(q=1)}$ as a function of $s$ for $k=1, n=1000$ and $R=1$.

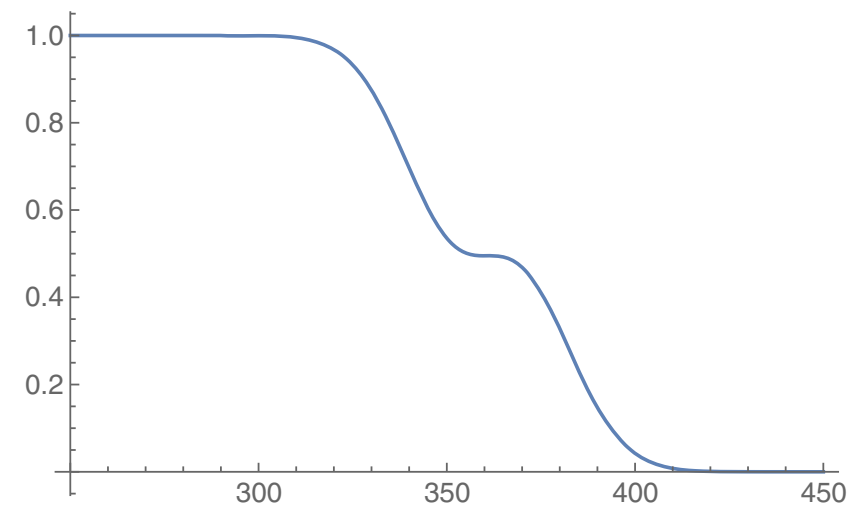

FIG. 6. Plot of $\lambda_{s}^{(q=1)}$ as a function of $s$ for $k=1, n=1000$ and $R=0.75$.

expects more steps around the transition region for the corresponding eigenvalues $\lambda$. In fact based on the observation that the $q$ th level states can be written, up to normalization, as $\hat{R}_{+}^{q}|L L L, n \rightarrow n+2 q\rangle$ one can argue that the wave functions will have $q$ nodes and the profile of the corresponding $\lambda$ will display $q$ distinct steps. A similar steplike pattern was observed in [15] for the higher Landau edge density functions for circular samples.

One can try to repeat the semiclassical analysis we did before for the first Landau level. Equation (87) can be written as

$$
\begin{aligned}
\lambda_{s} & =\frac{(n+3) !(n+2)}{s !(n+2-s) !} \int_{0}^{t_{0}} d t e^{F(t)}\left[t-\frac{s}{n+2}\right]^{2}, \\
F(t) & =(s-1) \log t+(n-s+1) \log (1-t) .
\end{aligned}
$$

The maximum of $F(t)$ occurs at $t^{*}=(s-1) / n$. Expanding $F(t)$ around $t^{*}$ we find that $e^{F}$ becomes a Gaussian function of narrow width centered around $t^{*}$. In fact,

$$
\left.\frac{d^{2} F}{d t^{2}}\right|_{t^{*}}=-\frac{n^{3}}{(n-s+1)(s-1)}
$$

Around the transition region the main contribution of the integral comes from the range of $s$ around $s^{*}$ such that $t^{*}=t_{0}$, namely

$$
t^{*}=\frac{s^{*}-1}{n}=t_{0} \quad \Rightarrow s^{*}=t_{0} n+1, \quad n-s^{*}+1=n\left(1-t_{0}\right) .
$$

We now evaluate the integral in (88) by expanding the integrand around $t_{0}$. In expanding $(t-s /(n+2))^{2}$ around $t_{0}$ we find that the large- $n$ contribution comes from the $\left(t-t_{0}\right)^{2}$ term. The constant and linear term in $t$ are suppressed by powers of $n$,

$$
\begin{aligned}
& \int_{0}^{t_{0}} e^{F(t)}\left(t-\frac{s}{n+2}\right)^{2} \\
& \sim e^{F\left(t_{0}\right)} \int_{0}^{t_{0}} \exp \left[-\frac{n}{2 t_{0}\left(1-t_{0}\right)}\left(t-t_{0}\right)^{2}\right]\left(t-t_{0}\right)^{2} \\
& =e^{F\left(t_{0}\right)} \frac{\sqrt{\pi}}{4 n \sqrt{n}}\left(2 t_{0}\left(1-t_{0}\right)\right)^{3 / 2}
\end{aligned}
$$

Substituting this in (88) and using Stirling's formula $n !=\sqrt{2 \pi n}(n / e)^{n}$, we find that

$$
\lambda_{s_{*}}^{(q=1)}=\frac{1}{2}
$$

independent of $t_{0}$ which is of course what is expected.

Although the semiclassical treatment above is sufficient to capture the value of $\lambda^{(q=1)}$ at the transition point, the evaluation of $H_{s}^{(q=1)}$ is more involved since it cannot be approximated by a simple Gaussian due to the steplike pattern for $\lambda^{(q=1)} . H_{s}^{(q=1)}$ will remain approximately flat in the steplike region, so higher derivatives around $s^{*}$ will be important to capture the correct behavior around the transition region. Figures 7 and 8 display the plots of $H_{s}^{(q=1)}$ around $\lambda=1 / 2$ based on the numerical evaluation

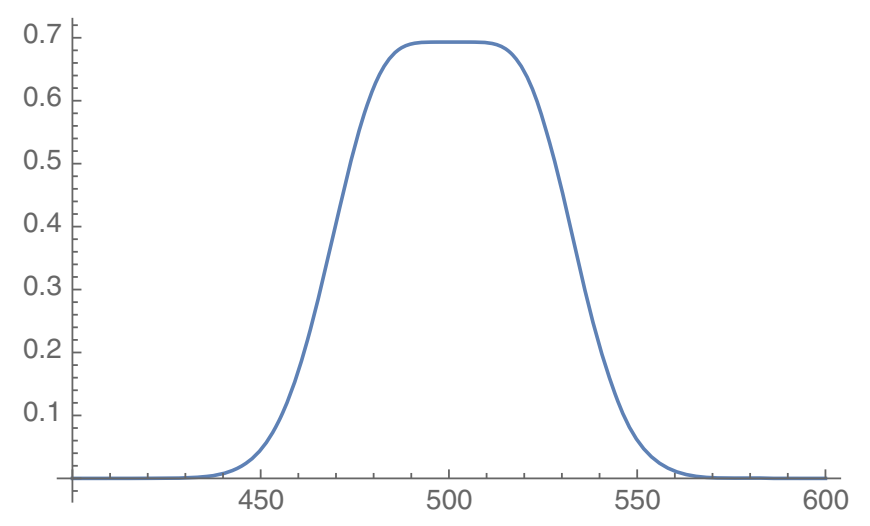

FIG. 7. Plot of $H_{s}^{(q=1)}$ as a function of $s$ for $n=1000$ and $R=1$. 


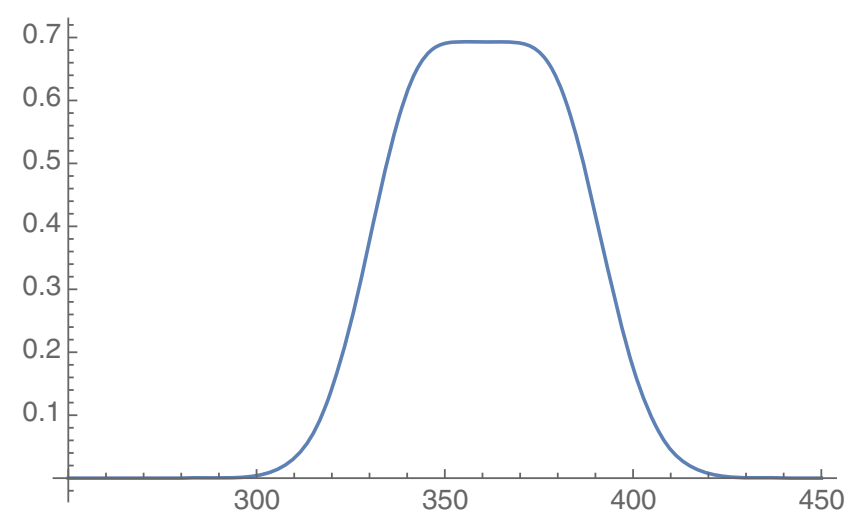

FIG. 8. Plot of $H_{s}^{(q=1)}$ as a function of $s$ for $n=1000$ and $R=0.75$.

of the exact expressions in (41) and (87). This clearly shows a deviation from the Gaussian distribution (see also Fig. 13). As a result the entropy for the first Landau level is larger than the entropy of the LLL even though the number of states are approximately the same at large $n$ $(n+1$ states for $q=0$ and $n+3$ states for $q=1)$. A numerical evaluation of the entropy shows that it obeys an area law and it gives

$$
S^{(q=1)}=1.65 S^{(q=0)} .
$$

When both $q=0$ and $q=1$ levels are filled, namely $\nu=2$, the situation is more involved as there are overlaps between the wave functions of different Landau levels. In particular,

$$
\begin{aligned}
\delta \lambda_{s, s^{\prime}}= & \int_{0}^{R^{2}} \Psi_{s}^{*(q=0)}(r) \Psi_{s^{\prime}}^{(q=1)}(r) d \mu \\
= & \delta_{s+1, s^{\prime}} \frac{(n+1) !}{s !(n-s) !} \sqrt{\frac{n+3}{(s+1)(n+1-s)}} \\
& \times \int_{0}^{R^{2}}\left[-\frac{(n+2) x^{s+1}}{x^{n+3}}+\frac{(s+1) x^{s}}{x^{n+2}}\right]
\end{aligned}
$$

The two-point correlator now is given by

$$
C\left(r, r^{\prime}\right)=\sum_{s=0}^{n} \Psi_{s}^{* 0}(r) \Psi_{s}^{0}\left(r^{\prime}\right)+\sum_{s=0}^{n+2} \Psi_{s}^{* 1}(r) \Psi_{s}^{1}\left(r^{\prime}\right)
$$

and

$$
\int C\left(r, r^{\prime}\right)\left(\begin{array}{c}
\Psi_{s}^{* 0}\left(r^{\prime}\right) \\
\Psi_{s+1}^{* 1}\left(r^{\prime}\right)
\end{array}\right) d \mu^{\prime}=\left(\begin{array}{cc}
\lambda_{s}^{0} & \delta \lambda_{s, s+1} \\
\delta \lambda_{s, s+1} & \lambda_{s+1}^{1}
\end{array}\right)\left(\begin{array}{c}
\Psi_{s}^{* 0}(r) \\
\Psi_{s+1}^{* 1}(r)
\end{array}\right)
$$

where $\lambda^{0}, \lambda^{1}$ are the eigenvalues we derived earlier for the lowest and first Landau level and $\delta \lambda$ is the overlap in (94).
There are $2 n+4$ eigenvalues for the two-point correlator given by $\lambda_{0}^{1}, \tilde{\lambda}_{s}^{ \pm}, \lambda_{n+2}^{1}$, where $s=0, \ldots, n$ and

$$
\tilde{\lambda}_{s}^{ \pm}=\frac{\lambda_{s}^{0}+\lambda_{s+1}^{1} \pm \sqrt{\left(\lambda_{s}^{0}-\lambda_{s+1}^{1}\right)^{2}+4\left(\delta \lambda_{s, s+1}\right)^{2}}}{2}
$$

The interesting feature here is that once both Landau levels are included the steplike pattern in the profile of $\lambda^{1}$ disappears. The profile of the new $\tilde{\lambda}^{ \pm}$resembles that of $\lambda^{0}$ but shifted with respect to $\lambda^{0}$; see Figs. 9 and 10. As a result the corresponding entropy per mode $\tilde{H}_{s}^{ \pm}$, where

$$
\tilde{H}_{s}^{ \pm}=-\tilde{\lambda}_{s}^{ \pm} \log \tilde{\lambda}_{s}^{ \pm}-\left(1-\tilde{\lambda}_{s}^{ \pm}\right) \log \left(1-\tilde{\lambda}_{s}^{ \pm}\right)
$$

are Gaussian distributions each centered around the value of $s$ for which $\tilde{\lambda}^{ \pm}=1 / 2$ as shown in Figs. 11 and 12 . Figure 13 shows a comparison between $H^{(q=0)}, H^{(q=1)}$ and $H^{(\nu=2)}$ which explains the differences in the values of the corresponding entropies, namely

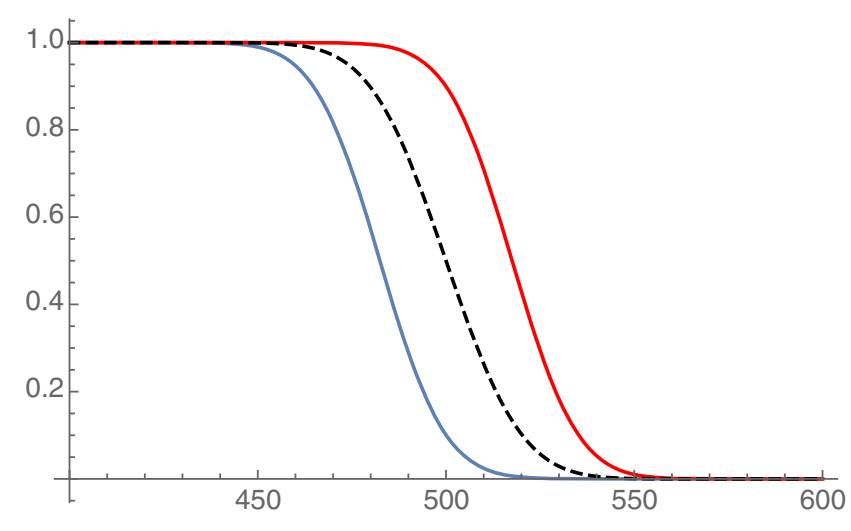

FIG. 9. Plots of $\tilde{\lambda}^{+}$(red to the right), $\tilde{\lambda}^{-}$(blue to the left) as functions of $s$ for $n=1000$ and $R=1$, compared to $\lambda^{0}$ (dashed, center).

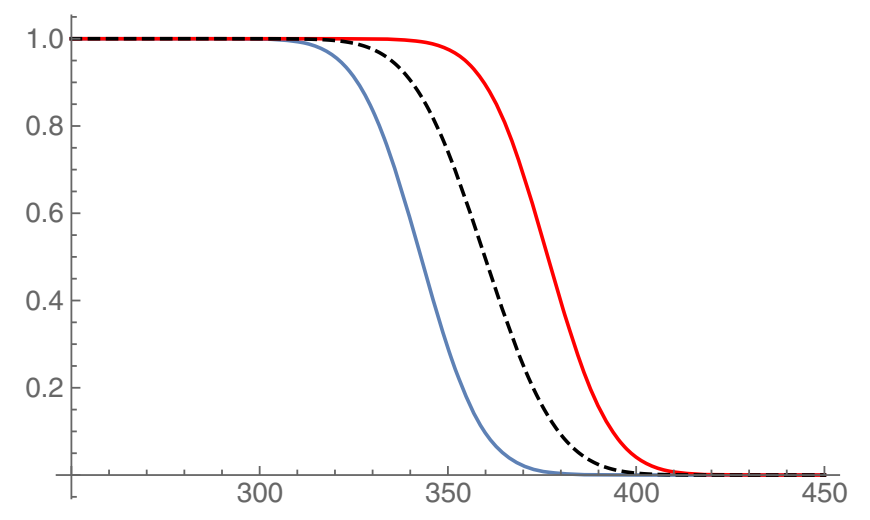

FIG. 10. Plots of $\tilde{\lambda}^{+}$(red to the right), $\tilde{\lambda}^{-}$(blue to the left) as functions of $s$ for $n=1000$ and $R=0.75$, compared to $\lambda^{0}$ (dashed, center). 


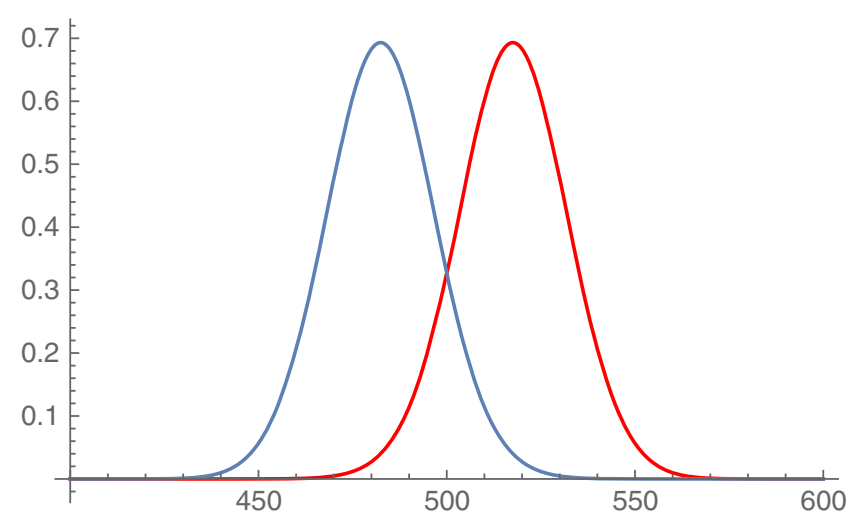

FIG. 11. Plots of $\tilde{H}^{+}$(red to the right) and $\tilde{H}^{-}$(blue to the left) as functions of $s$ for $n=1000$ and $R=1$.

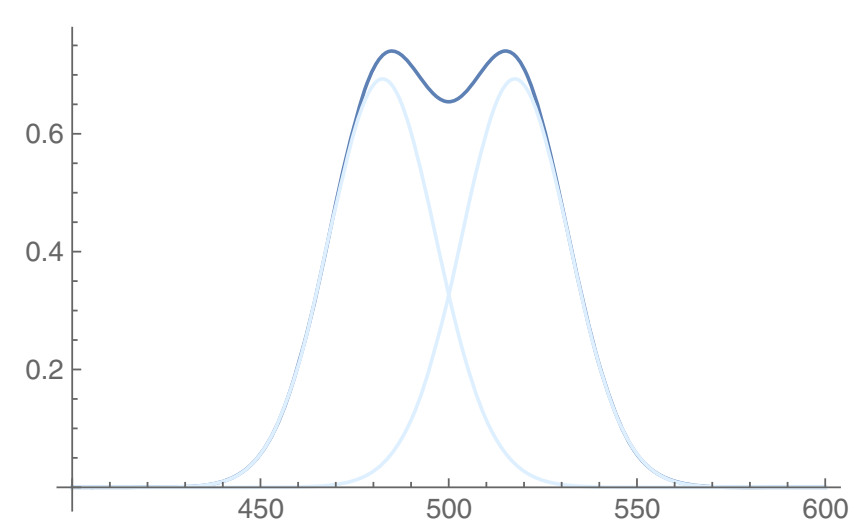

FIG. 12. Plot of $\tilde{H}^{+}+\tilde{H}^{-}$as function of $s$ for $n=1000$ and $R=1$.

$$
S^{(\nu=2)}>S^{(q=1)}>S^{(\nu=1)}
$$

A numerical evaluation of the entropy for the $\nu=2$ case gives

$$
S^{(\nu=2)}=1.76 S^{(\nu=1)} .
$$

This agrees with the result in [2].

\section{DISCUSSION}

In this paper we have analyzed the entanglement entropy for fully filled $\nu=1$ higher-dimensional quantum Hall effect on $\mathbb{C P}^{k}$ for Abelian and non-Abelian magnetic fields. The analytical calculation is based on a semiclassical

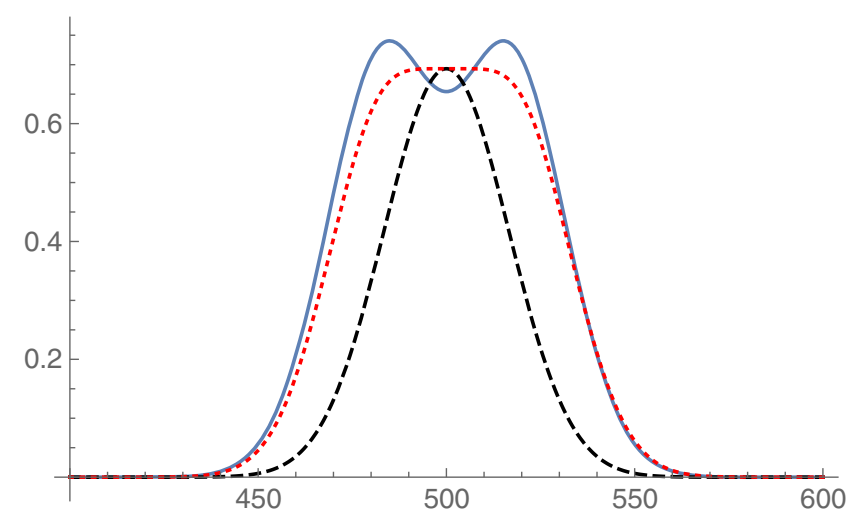

FIG. 13. Plots of $H^{(\nu=1)}$ (black, dashed), $H^{(q=1)}$ (red, dotted) and $H^{(\nu=2)}$ (blue, solid) as functions of $s$ for $n=1000$ and $R=1$.

analysis and we have showed that the entropy satisfies the area law. In fact the entropy as expressed in terms of a phase-space entangling surface area has the same proportionality constant for all higher dimensions irrespective of the Abelian or non-Abelian nature of the background magnetic field. It will be interesting to see if a similar universal formula can be obtained for higher Landau levels.

In the presence of edge degrees of freedom the entanglement entropy for the two-dimensional integer quantum Hall effect develops subleading logarithmic contributions [6] It has been shown in the two-dimensional $\nu=1$ quantum Hall effect that when the edge boundary intersects the boundary of the entangling surface there is an additional logarithmic contribution whose coefficient is determined by the central charge of the gapless edge modes [7,8]. In the context of higher-dimensional quantum Hall effect we have previously analyzed the analogs of higher-dimensional chiral Abelian and non-Abelian droplets, the edge spectrum and corresponding effective actions [11]. It would be interesting therefore to extend the analysis of the entanglement entropy to these cases where the entangling surface and edge boundary overlap in higher dimensions and calculate the corresponding subleading corrections to the area law for the entanglement entropy.

Similar considerations for higher Landau levels in both two and higher dimensions are also worth pursuing.

\section{ACKNOWLEDGMENTS}

I thank V. P. Nair for helpful discussions. This research was supported in part by the National Science Foundation Grant No. PHY-1915053 and by PSC-CUNY grants. 
[1] A. Kitaev and J. Preskill, Phys. Rev. Lett. 96, 110404 (2006); M. Levin and X. G. Wen, Phys. Rev. Lett. 96, 110405 (2006).

[2] I. D. Rodriguez and G. Sierra, Phys. Rev. B 80, 153303 (2009); J. Stat. Mech. (2010) P12033.

[3] A. Sterdyniak, A. Chandran, N. Regnault, B. A. Bernevig, and P. Bonderson, Phys. Rev. B 85, 125308 (2012).

[4] J. Dubail, N. Read, and E. H. Rezayi, Phys. Rev. B 85, 115321 (2012); 86, 245310 (2012).

[5] A. Petrescu, H. F. Song, S. Rachel, Z. Ristivojevic, C. Flindt, N. Laflorencie, I. Klich, N. Regnault, and K. Le Hur, J. Stat. Mech. (2014) P10005.

[6] H. Li and F. D. Haldane, Phys. Rev. Lett. 101, 010504 (2008).

[7] B. Estienne and J.-M. Stephan, Phys. Rev. B 101, 115136 (2020).
[8] P.-G. Rozon, P.-A. Bolteau, and W. Witczak-Krempa, arXiv: 1911.1128.

[9] L. Charles and B. Estienne, Commun. Math. Phys. 376, 521 (2020).

[10] D. Karabali and V.P. Nair, Nucl. Phys. B641, 533 (2002).

[11] D. Karabali and V. P. Nair, Nucl. Phys. B679, 427 (2004); B697, 513 (2004).

[12] D. Karabali, Nucl. Phys. B726, 407 (2005); B750, 265 (2006); D. Karabali and V. P. Nair, Phys. Rev. D 94, 024022 (2016).

[13] D. D. M. Haldane, Phys. Rev. Lett. 51, 605 (1983).

[14] V. P. Nair, Phys. Rev. D 101, 125021 (2020).

[15] G. V. Dunne, Int. J. Mod. Phys. B 08, 1625 (1994). 